\title{
Round 2: Flight, Floating, and Mars Courses
}

\author{
Sarah J. Desotell \\ Department of Physics, Ripon College \\ Ripon, Wisconsin
}

\begin{abstract}
Exploring Mars" and "Flight and Floating" are new courses that have been integrated into the Ripon College curriculum. "Exploring Mars" was developed for non-science major students to increase their general scientific interest through topics of space exploration and space science. "Flight and Floating" was designed for students with physics, math, and engineering interests, combining experimental design and mathematical modeling to study drag, lift, thrust, and buoyancy.
\end{abstract}

\section{Overview}

The goal of this two-year project was to develop new undergraduate courses for a diverse audience of students, in topics of space exploration and aerospace studies. The first course, "Exploring Mars," introduced first-year students to the science of space exploration through topics such as space travel and energy technology, terraforming and colonizing Mars, and current events in space discoveries. One objective of this course was to increase general scientific interest in students who were not planning to major in the natural sciences. The second, two-part course sequence, "Introduction to Flight" and "Flight and Floating," combined mathematical modeling with experiments to observe and analyze floating and flying objects. The flight course sequence was intended for students with experience in physics and mathematics, and was especially suited for students planning to pursue further studies and/or careers in engineering and aerospace science. Each new course has been offered twice at Ripon College. With some revisions in course activities and content, "Exploring Mars" will be offered for a third time in the fall of 2011, and "Flight and Floating" will likely be offered on a two or three-year rotation in the future. This paper describes several course activities and provides examples of the course development that has occurred over the past two years.

\section{Exploring Mars: Course Design and Development}

Through labs, field trips, lecture, and other activities, first-year students in "Exploring Mars" covered a wide variety of topics in space science. The course largely focused on Mars (climate, weather, geography, rover and orbiter missions, life and water, terraforming and colonization), but also engaged students in broader topics such as energy technology (solar cells, fuel cells), space travel (rockets, magnetic sails), space ethics (space garbage, animals in space, terraforming), and current events in space exploration. The course was first taught in the fall of 2009 with an enrollment of twenty students, clustered with another first-year course entitled "Plants in Space." A detailed description of the original course curriculum, goals, and activities is provided in the 2010 WSGC Conference Proceedings (Desotell, 2010).

This project has been supported by the Wisconsin Space Grant Consortium Higher Education Incentives Program and by Ripon College. Special thanks to Skip Wittler, Ripon College Professor of Biology, for the development of the "Plants in Space" course. 
During the fall of 2010, "Exploring Mars" was offered a second time to twenty first-year students, maintaining the same general course structure and content, with minor revisions in assignments and activities. For example, a solar cell lab was added to the 2010 curriculum. In this lab activity, accompanied by a lecture on solar cell operation and applications, each group of three or four students was given a solar-powered vehicle, and calculated the minimum intensity and power required for the vehicle to operate, based on the distance and power of a given light source. Students could then confirm whether or not their solar vehicle could operate on Mars. This lab followed a few weeks behind the "Power of the Sun" lab, where students were first introduced to concepts and mathematical relationships involving intensity and power and calculated the intensity of the sun on Mars. Typically, in this non-major course of first-year students, there is a broad range of mathematical background and skill among the students. Using the same mathematical relationships to study different systems can be a helpful way to increase students' comfort level with mathematical analysis. On the other hand, a time-consuming 2009 lab, "Heating the Surfaces of Earth and Mars" was eliminated from the course in 2010. The lab was focused on temperature differences of materials with unequal specific heats, and produced large amounts of data for students to analyze. Without including differences in atmosphere and distance to the sun, the lab is not a good representation of Earth/Mars temperature differences.

Additional course revisions in 2010 devoted more attention to current events in space science by assigning students to find, review, and share articles related to space exploration. Also, a more clearly defined final course project was assigned, requiring students to develop a habitat for humans on Mars including plans for power, water, shelter, and agriculture. Student presented the group project in a formal paper as well as in poster session with the students from "Plants in Space," who presented habitat designs as well. This assignment was a suitable final project, challenging students to combine ideas from the entire semester to produce a complete proposal, with infinite possibilities inviting creative solutions. Student evaluations for the 2009 and 2010 Mars courses were largely positive and reflected that many students were interested and engaged in the material and enjoyed a wide range of course activities, although some students commented that the course was repetitive and slow-paced.

\section{Round 3: Mars in 2011}

"Exploring Mars" will be offered for a third time in the fall of 2011, with the course goals as listed in Table 1. Although the course will retain similar structure and activities to previous years, revisions will continue to be made to create effective teaching strategies and assignments, and to keep up with current events and resources. For example, the second lab of the semester, "How High Can You Jump on Mars?" students take measurements of Earth-bound jump heights, calculations of take-off velocity (knowing the rate of gravitational acceleration on Earth), and the equivalent jump height on Mars. This year, the lab will be extended to include calculation of the gravitational acceleration on Mars, for use in jump calculations. Determination of gravitational acceleration will require students to find the sun's mass and the radius of Mars using external sources, and will be an exercise in unit analysis and conversions and increase students' familiarity with scientific notation. The 2011 presentation assignment has also been modified from previous years. In the past, students presented movie reviews with scientific analyses using fictional movies set in outer space. During the fall of 2011, the presentation assignment will instead focus on real missions to Mars; Students will focus on mission goals, scientific 
instrumentation, challenges, failures, and successes. The assignment will be introduced near the beginning of the course when many students are just becoming acquainted with Mars.

Table 1: Course goals for "Exploring mars" in 2011

- Develop skills in written and oral communication and quantitative analysis

- Gain experience in experimental design and measurement techniques

- Study the climate of Mars with comparisons to Earth's climate

- Understand the physics and applications of various energy technologies

- Understand past and recent evidence of life and water on Mars

\section{Flight and Floating: Course Design and Development}

"Introduction to Flight" was a semester-long course immediately followed by a three-week "Flight and Floating" Maymester. The course sequence focused on flying and floating objects (rockets, parachutes, kites, balloons, airplanes, birds, and many more) through experimental and mathematical analysis, field trips, and guest speakers. The sequence was designed for students with experience in physics and math, and was especially suited for students interested in pursuing careers in the aerospace and aeronautics industries or in engineering. The introductory course met on a weekly basis and often consisted of a conceptual and mathematical introduction to a force (drag, lift, thrust, buoyancy), followed by a lab activity that required measurements and analysis. During the second course of the sequence, "Flight and Floating" students applied these concept and models to design and analyze more complex systems demonstrating lift, drag, gravity, buoyancy, and thrust.

Rockets, for example, were studied extensively during the course sequence through a variety of experiments, using several different modeling and analysis techniques. During "Introduction to Flight," the rocket unit began with a basic explanation of rocket thrust resulting in forward motion. Students launched Alka-Seltzer ${ }^{\circledR}$ rockets and balloon rockets to demonstrate the concept. This was followed by a derivation describing the time-varying acceleration and velocity of a rocket in flight. The derivation begins with the change in momentum, $\Delta p$, of the system as a mass of burned fuel, $\Delta m$, leaves the rocket with an exhaust velocity $v_{e}$ (relative to the rocket). Figure 1 shows a rocket with mass $M$ and velocity $V$ at some initial time $t$. A short time later $(t+$ $\Delta t$ ), the rocket velocity and mass have changed to $V+\Delta V$ and $M-\Delta m$, respectively. The total change in momentum, observed from a stationary reference frame (the ground) is given by equation 1 . The exhaust velocity relative to the ground, $v_{e, g}$, is given by equation 2 (where $v_{e}$ is a positive quantity), and the total change in momentum is simplified to equation 3 , assuming that the product $\Delta m \bullet \Delta V$ is negligible compared to other terms for small time steps.

$$
\begin{aligned}
& \Delta p=\left\lfloor(M-\Delta m)(V+\Delta V)+\Delta m \cdot v_{e, g}\right\rfloor-M \cdot V \\
& v_{e, g}=V-v_{e} \\
& \Delta p=M \cdot \Delta V-\Delta m \cdot v_{e}
\end{aligned}
$$


When equation 3 is applied in Newton's Second Law $\left(F_{n e t}=\Delta p / \Delta t\right.$, where $F_{n e t}$ is the net external force), equation 4 is derived, including rocket acceleration, $a=\Delta V / \Delta t$, and fuel burn rate, $m=\Delta m / \Delta t$. The governing equations simplify to $F_{n e t}=M \cdot a$ when the fuel is exhausted and rocket mass becomes constant.

$$
\sum F=M \cdot a-\dot{m} \cdot v_{e}
$$

Figure 1: The mass and velocity of a rocket change as fuel is exhausted.

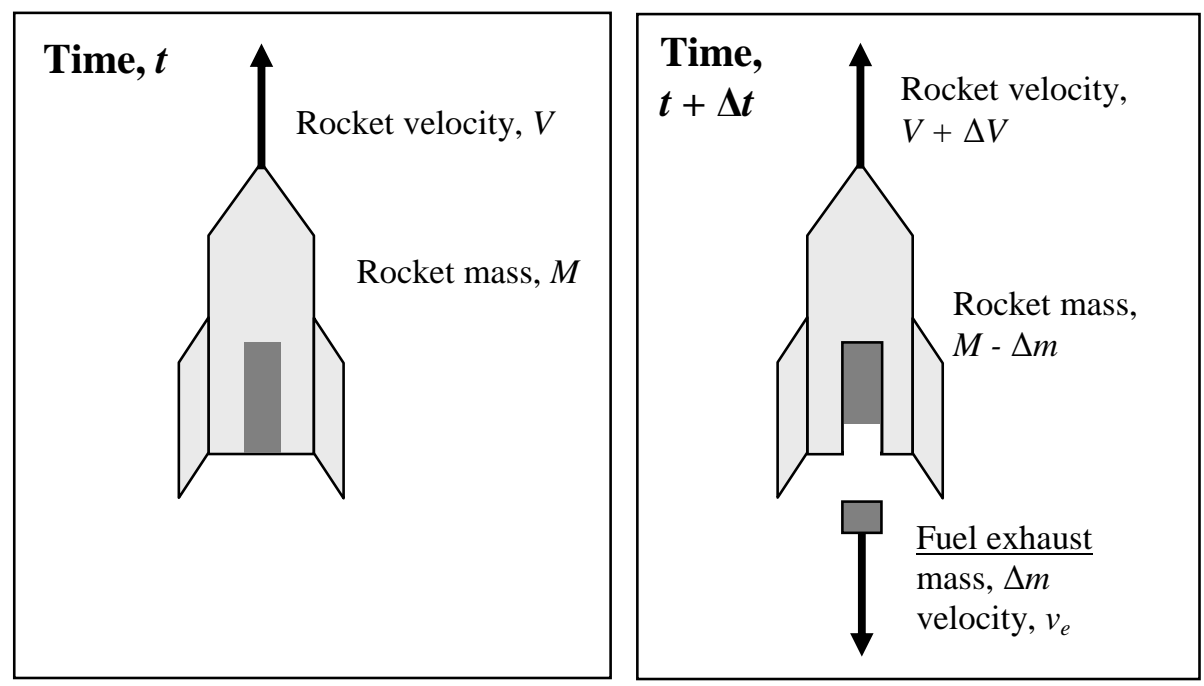

The rocket equation was applied to analyze several different systems during the two-course sequence. During the introductory course, students programmed the rocket equation in a Microsoft ${ }^{\circledR}$ Excel spreadsheet with a simple numerical scheme including forces of drag and gravity, to model the motion of pre-constructed solid-fuel Estes ${ }^{\circledR}$ rockets. Although many variations of this experiment could be executed, in this case, students combined experimental data with modeling assumptions to determine the average exhaust velocity, $v_{\mathrm{e}}$, of a rocket motor. To do so, the initial and final rocket masses were measured before and after the launch, respectively, and the average fuel burn rate was calculated using the total mass difference (the amount of fuel burned) and the total burn time, taken from Estes ${ }^{\circledR}$ reported data or using experimental measurements of burn time. Drag force parameters such as projected rocket area and air temperature were measured before the launch, and a drag coefficient was estimated in the $0.3-0.5$ range (nosecone.com). Finally, the apogee of the rocket was measured during flight using an altimeter carried within the rocket, and by students measuring the angle above the horizon at apogee from a known horizontal distance. With these parameters and measurements, each student developed a numerical model calculating time-varying mass, acceleration, velocity, and altitude of the rocket. Students determined the unknown exhaust speed by matching the numerically predicted apogee to the launch results.

During the Maymester course, "Flight and Floating," rockets were analyzed even further: Student built their own solid fuel rocket from an Estes ${ }^{\circledR}$ kit (skill level 2 or higher), and modified their rocket to include an electronics bay housing an altimeter. Each student chose one 
parameter to vary among launches, such as engine strength, total weight, or fin design. Each rocket was launched several times for each set of experimental conditions, and altimeters returned apogee measurements upon recovery. Students were introduced to RockSim rocket modeling software during this project, and developed RockSim models of the rockets they had constructed. Apogee date from the altimeters was compared to RockSim predictions, with good agreement in most cases. One altimeter also returned measurements of top speed and apogee time, which were compared to RockSim modeling predictions. The last rocket system studied during the Maymester was compressed air-water rockets. Students constructed simple rockets by attaching homemade fins and nose cones to one-liter or two-liter plastic bottles, and by adjusting the weight and pressure distributions to ensure stable flight. The rockets were launched using a custom-built launching mechanism and each flight was video recorded for analysis purposes. Students used an additional analysis tool, Logger Pro video analysis software, to determine variations in position, velocity, and acceleration of the water rocket. Although a numerical model was not applied to the water rockets, the time-dependent altitude profiles were compared to the experimental and modeling results of Gommes (Gommes, 2010), who presented a numerical model including thermodynamic analysis and other complex factors, attempting to predict the performance of pressurized water rockets.

Additional activities from the original course series are described in the 2010 Wisconsin Space Conference Proceedings (Desotell, 2010). In 2010, when "Introduction to Flight" was first offered, eight physics students enrolled in the introductory course and six of these students completed the 2010 Maymester. In 2011, eight students enrolled in the introductory course (including three physics majors, one math major, and four students with little experience in math or physics). Three physics majors completed the 2011 Maymester course. Because of the broad range of skill level in the 2011 class, two complex mathematical modeling assignments from 2010 were eliminated (modeling horizontal balloon rockets and pressure- and temperaturedependent high-altitude balloon calculations). Instead, in 2011, an oral presentation assignment was created. Examples of presentation topics chosen by the students included albatross flight, breaking the sound barrier, and hot air balloons, to name a few.

Finally, the course sequence concluded with unique, student-designed experiments that relied on helium balloons ( 3-foot-diameter) to carry experimental payloads to "high" altitudes. In 2011, the projects focused on 1) relating drag coefficients to parachute shape, 2) comparing the energy dissipation of a superball on different impact surfaces, and 3) temperature and heat transfer variations with altitude. However, wind speeds of 15-20 mph and $50 \mathrm{mph}$ gusts persisted for four days during the course, and the three balloon experiments were moved indoors to a gymnasium where the balloon height was limited to about 30 feet. The temperature experiment was modified for the gymnasium to detect a temperature gradient between floor and ceiling in the indoor facility. Although altitude was severely limited within the gymnasium, the balloon experiments were well designed and executed, and students learned about the need for flexibility in planning. In fact, the controlled conditions and absence of wind made the experimental procedures run more smoothly than would have been possible outdoors. Even when problems occurred, troubleshooting was relatively easy under the calm, indoor conditions of the gym. In the future, indoor facilities might be used to work through and refine experimental procedures before conducting outdoor experiments. 


\section{Round 3: Future Flight and Floating}

"Introduction to Flight" and "Flight and Floating" provided unique opportunities for students to engage in aerospace science. The course will likely be offered on a two or three-year rotation based on student interest and faculty course loads. The ideal enrollment for "Flight and Floating" is 6-10 students, and the course will continue to be designed for students with some experience in math and physics. In the future, the course sequence will likely be combined into one 3- or 4credit Maymester course (17-19 days long instead of 14) without the required pre-requisite semester-based portion of the course. This combined structure will improve continuity in the subject matter and there will be less need for repetition, compared to the two-course sequence. With the combination of the two courses into one longer Maymester course, more theoretical background will be incorporated into the typical Maymester day and more time will be needed for students to develop their modeling tools during the Maymester sessions. One or more lab activities will be eliminated to meet time constraints. Several experiments will be repeated next time the course is offered, such as determining wind speed by flying a kite, rocket launch and analysis, determining drag coefficients of parachutes, and an open-ended helium balloon project.

\section{Summary}

New courses on space exploration and aerospace science have been successfully integrated into the curriculum at Ripon College. "Exploring Mars" has been completed by 39 first-year students over the past two years, bringing the excitement of space exploration to the classroom through a variety of activities. Many of the students enrolled in the course did not intend to major in the sciences, but now have an increased general interest in space science as a result of this course. Student feedback has been largely positive and with on-going course revisions, "Exploring Mars" will be offered with "Plants in Space" for a third time during the fall of 2011. "Introduction to Flight" has been completed by 16 students over the past two years and 9 of these students completed the complimentary course, "Flight and Floating." This course sequence has been extremely successful in introducing students to aerospace science and in the development of experimental design and measurement skills through experiments, modeling, field trips, and other activities. Although geared towards students with interests in math, physics, and engineering, the courses have been accessible to students with less experience in these subject areas. "Flight and Floating" is likely to be offered again in 2013 and then on a two or three-year rotation, reduced to one intense Maymester course rather than a longer two-course sequence. "Exploring Mars" and "Flight and Floating" are unique, exciting, and valuable additions to the Ripon College curriculum.

\section{References}

Desotell, Sarah J. Flight, Floating, and Mars; New Courses at Ripon College. Proceedings of the 20th Annual Wisconsin Space Conference: Dawn of a New Age. Unpaginated. August 19-20, 2010, UW Sheboygan, Sheboygan, WI.

Drag Coefficient of Various Shapes, Ford Motor Co. and the National Research Council of Canada, <http://www.nosecone.com/table.htm, accessed May 2011>.

Gommes, Cedric J. A more thorough analysis of water rockets: Moist adiabats, transient flows, and inertial forces in a soda bottle. Am. J. Phys. 78 (3), 236-243, 2010. 